\section{Efeito da acupuntura sobre dinamometria manual e a resistência muscular localizada de membros superiores em praticantes de exercício resistido*}

\section{Effect of acupuncture on hand dinamometry and muscular localized resistance of practitioners upper limbs in resistance exercise}

\section{Resumo}

Estudo analítico transversal controlado não pareado que contou Déborah de Oliveira Castro Nataly Mitie Natsume Moriya ${ }^{3}$ Hugo Alves de Sousa ${ }^{4}$ José Roberto P.de Godoy ${ }^{5}$

* Recebido em: 11/11/2014.

Aprovado em: 28/05/2015.

1 Centro Universitário de Brasília - Faculdade de Fisioterapia - UniCEUB. email: andreiyuri7@ gmail.com.

2 Centro Universitário de Brasília - Faculdade de Fisioterapia - UniCEUB. email: deboraah_7@ hotmail.com.

3 Centro Universitário de Brasília - Faculdade de Biomedicina - UniCEUB. email: natymitie@ hotmail.com.

4 Universidade de Brasília - Faculdade de Medicina - Área de Morfologia, Mestre em Ciências da Saúde pela Universidade de Brasília - UnB, Doutorando em Ciências Médicas pela Universidade de Brasília - UnB. email: hugoanatomia@gmail.com.

5 Universidade de Brasília - Faculdade de Medicina - Área de Morfologia, Mestre e Doutor em Ciências da Saúde. email: profbetogodoy@ gmail.com.

\begin{abstract}
com 14 participantes do sexo masculino, selecionados por conveniência com idades entre 20 e 25 anos, praticantes de exercício resistido, divididos em dois grupos não pareados, sendo: um grupo sem intervenção por acupuntura (GC) e o outro grupo com intervenção com acupuntura (GE). O objetivo do presente estudo consistiu em avaliar o efeito da acupuntura sobre a resistência muscular localizada de membros superiores e a força de preensão palmar em praticantes de exercício resistido. Com relação à resistência muscular localizada, os resultados apontam aumento do número de repetições para os dois grupos, porém, não estatisticamente significativos e para força de preensão palmar, aumento para o GE e diminuição para o GC, também não estatisticamente significativas. Conclui-se que a pesquisa sobre a acupuntura, apresentada neste artigo, não se demonstrou eficaz para aumentar o rendimento dos sujeitos no teste de resistência muscular localizada e da força de preensão palmar.
\end{abstract}

Palavras-chave: Acupuntura. Resistência Muscular Localizada. Força de Preensão Palmar. Exercício Resistido.

\begin{abstract}
Controlled cross-sectional analytical study unpaired that featured 14 male participants, selected by aged between 20 and 25 years, practitioners of resistance exercise, divided into two unpaired groups: one group without intervention by acupuncture (GC) and the other group with intervention acupuncture (GE). The aim of this study was to evaluate the effect of acupuncture on muscular endurance of upper limbs and the handgrip strength in practitioners of resistance exercise. With respect to muscular endurance, the results indicate an increase in the number of repetitions for the two groups, but not statistically significant and to grip strength, increase and decrease in GE and GC respectively, also not statistically significant. It is concluded that acupuncture in this study did not prove to be effective to increase the yield of the subjects in the muscular endurance test and grip strength.
\end{abstract}

Keywords: Acupuncture. Muscular Localized Resistance. Grip Strenght. Resistance Exercices. 


\section{Introdução}

A resistência muscular configura uma das medidas de registro da capacidade funcional para um músculo ou grupamento muscular. A melhora na resistência muscular é importante, pois algumas reduções nas atividades funcionais dos adultos parecem estar associadas à incapacidade do indivíduo em conservar esforços repetitivos, fundamentais para continuar atividades da vida diária (ARAGÃO et al., 2002). Dessa forma, a resistência muscular localizada (RML) constitui parâmetro relevante da função neuromuscular que tem recebido pouca atenção da literatura (BEMBEN, 1998), mas que pode oferecer medida mais prática da função muscular comparada às atividades da vida diária (BEMBEN, 1996).

A mensuração da força de preensão permite investigar de forma objetiva a integridade funcional dos membros superiores. Contudo não é limitada apenas a ele, pois apresenta diversas aplicações clínicas diferentes, sendo, por exemplo, indicador da força total do corpo, e nesse sentido pode ser também aplicado em testes de aptidão física (DURWARD et al., 2001).

A eficácia da acupuntura sobre as variáveis da performance física de praticantes de atividades físicas saudáveis e de alto rendimento não são claras. Contudo, alguns autores têm afirmado que a acupuntura promove melhora do desempenho físico e da capacidade desportiva (PELHAM et al., 2001; JAUNG-GENG et al., 1995). Embora os mecanismos subjacentes sejam amplamente desconhecidos, há apontamentos na literatura que revelam que a acupuntura estimula modificações na excitabilidade da via motora, podendo fornecer adequada base fisiológica para explicar a ligação entre a acupuntura e o desempenho motor (LO et al., 2005). Hubscher et. al (2010) observaram que a intervenção por acupuntura em uma sessão foi capaz de induzir aumento na força isométrica no quadríceps femoral em atletas recreativos.

No entanto não há evidências suficientes publicadas na literatura a favor ou contra aos efeitos da acupuntura sobre a performance muscular. A estimativa da eficácia é dificultada por diferentes técnicas de acupuntura, condições de controle, procedimentos dos testes múltiplos, limitações nos tamanhos de amostras e qualidade do estudo metodológico (WHI- estudo foi avaliar o efeito da acupuntura sobre a resistência muscular localizada de membros superiores e a força de preensão palmar em praticantes de exercício resistido.

\section{Material e métodos}

\subsection{Amostra}

O presente estudo pode ser caracterizado como longitudinal prospectivo, com 14 participantes. Foram selecionados por conveniência, no campus do Centro Universitário de Brasília - UniCEUB, 14 voluntários do sexo masculino, com idade entre 20 e 25 anos, todos praticantes de exercício resistido. Os participantes foram divididos em dois grupos não pareados, sendo um grupo sem intervenção por acupuntura (GC) e o outro grupo com intervenção com acupuntura (GE).

Os critérios de inclusão foram: ser considerado fisicamente ativo de acordo com o International Physical Activity Questionnaire (IPAQ), versão curta, e assinar devidamente o Termo de Consentimento Livre e Esclarecido (TCLE). Já os critérios de exclusão consistiram em não apresentar o TCLE devidamente assinado e apresentar afecções osteomusculares que impedissem a realização das atividades propostas no estudo.

\subsection{Procedimentos}

A coleta de dados foi realizada de duas formas distintas. Os participantes do GE submeteram-se a um teste de força, no qual deveriam realizar três medidas de preensão com um dinamômetro palmar. O intervalo estabelecido entre as medidas de FPP foi de 1 minuto, conforme as recomendações da Sociedade Americana de Terapeutas da Mão (SATM). Seguido do teste de preensão palmar, os participantes foram submetidos ao teste de resistência muscular localizada (RML), o qual consistia de o máximo de repetições de flexão de braço que o sujeito conseguisse executar durante 1 minuto. Logo após o teste de RML, os participantes foram convidados a deitarem na maca em decúbito dorsal. Após 1 minuto nesse posicionamento, foram inseridas as agulhas nos acupontos IG11, ID8, TA11, TA15 e P1 bilateralmente, por 20 minutos. Após a intervenção por acupuntura, os participantes foram instruídos a repetir os testes anteriormente relatados.

Os participantes do GC realizaram os mesmos tes- 
tes do GE, porém não houve aplicação da acupuntura, ou seja, os sujeitos ficaram em repouso durante 20 minutos após os testes. Logo após o repouso, repetiram os testes de FPP e RML.

\subsection{Teste de preensão palmar}

Foi realizada a avaliação da força de preensão palmar (FPP) do membro dominante, utilizando-se o dinamômetro palmar SAEHAN`. O dinamômetro é composto por duas barras de aço ligadas juntas que ao serem apertadas se dobram, provocando alteração na resistência dos aferidores, sendo essa diretamente proporcional à força de preensão exercida pela mão (MOREIRA et al., 2001; MOREIRA et al., 2003). Os participantes foram orientados a permanecer sentados seguindo a posição padronizada da SATM, ou seja, os quadris e joelhos devem estar fletidos a $90^{\circ}$, com ombros aduzidos, cotovelo também a $90^{\circ}$ e antebraço em posição neutra (FESS, 1992). Estando nesta posição, foram orientados a apertar o dinamômetro após comando verbal durante 5 segundos. $\mathrm{O}$ teste foi repetido três vezes com intervalos de 1 minuto entre cada preensão.

\subsection{Teste de Resistência Muscular Localizada}

O procedimento adotado consistiu na flexão dos cotovelos até que estes alcancem o nível dos ombros, seguido do retorno à posição inicial. Os movimentos são contados durante o período máximo de 1 minuto ou até atingir a exaustão. Na presente pesquisa, adotou-se protocolo com duas anilhas de $10 \mathrm{~kg}$, onde os sujeitos deveriam movimentar a barra com os pesos de acordo com o compasso de um metrônomo calibrado a $4 \mathrm{bpm}$. Ao término do teste de RML, houve intervalo de recuperação de 2 minutos para restauração da frequência cardíaca normal.

\subsection{Acupuntura}

Foram utilizados cinco acupontos bilateralmente, sendo eles IG11 (depressão na extremidade externa da prega de flexão do cotovelo), ID8 (na depressão óssea da face posteromedial do cotovelo, na incisura do nervo cubital), TA12 (no dorso do braço, aproximadamente na metade do comprimento do úmero) TA15 (depressão superior da espinha escapular) e P1 (região superolateral torácica, no primeiro espaço intercostal, ligeiramente medial ao limite inferior do processo coracoide clavicular) (MACIOCIA, 1996) (Quadro 1). Os acupontos fo- ram punturados na ordem acima descrita e as agulhas $(0.25 \mathrm{~mm} \times 30 \mathrm{~mm}$, marca Han Sol') inseridas e estimuladas até que o paciente relatasse o De Qi, ou seja, a sensação de dormência, peso, tensão, provocada pelas agulhas (LIAN et al., 2005). Os indivíduos ficaram sentados em repouso com a aplicação das agulhas durante 20 minutos. Após a remoção destas, permaneceram em repouso por 2 minutos antes da repetição dos testes.

Quadro 1 - Pontos de acupuntura utilizados.

\begin{tabular}{|l|l|l|}
\hline \multicolumn{1}{|c|}{ Nome } & \multicolumn{1}{|c|}{$\begin{array}{c}\text { Indicações (relacionadas aos } \\
\text { membros superiores) }\end{array}$} & \multicolumn{1}{c|}{$\begin{array}{c}\text { Principais funções } \\
\text { energéticas }\end{array}$} \\
\hline $\begin{array}{l}\text { IG 11 } \\
\text { (Quchi) }\end{array}$ & $\begin{array}{l}\text { Cervicobraquialgia, inflamação e dor } \\
\text { em cotovelo e braço, desequilíbrio } \\
\text { motor das extremidades superiores, } \\
\text { dor artrítica de membros superiores. }\end{array}$ & $\begin{array}{l}\text { Harmoniza o Qi } \\
\text { Fortalece e resfria o Xue. } \\
\text { Fortalece os tendões e } \\
\text { articulações. }\end{array}$ \\
\hline $\begin{array}{l}\text { ID 8 } \\
\text { (Xiaohai) }\end{array}$ & $\begin{array}{l}\text { Dor em ombro, nuca, pescoço e região } \\
\text { escapular; inflamação e dores em } \\
\text { cotovelo, braço e antebraço; neuralgia } \\
\text { e paralisia do nervo ulnar. }\end{array}$ & $\begin{array}{l}\text { Relaxa músculos e tendões. } \\
\text { Ativa a circulação de Xue. }\end{array}$ \\
\hline $\begin{array}{l}\text { TA 12 } \\
\text { (Xiaoluo) }\end{array}$ & $\begin{array}{l}\text { Rigidez cervical; ombro congelado/ } \\
\text { dor em obro, pescoço e costas; } \\
\text { enfraquecimento motor e dor no braço. }\end{array}$ & $\begin{array}{l}\text { Regula e promove } \\
\text { circulação do Xue. }\end{array}$ \\
\hline $\begin{array}{l}\text { P1 } \\
\text { (Zhongfu) }\end{array}$ & $\begin{array}{l}\text { Escapulalgia, braquialgia, cervicalgia, } \\
\text { dor e rigidez do pescoço, dor na nuca, } \\
\text { rigidez do ombro, dor em ombro e } \\
\text { cotovelo, dor na fossa supraclavicular, } \\
\text { tendinite supraespinal. }\end{array}$ & $\begin{array}{l}\text { Alivia rigidez muscular. } \\
\text { Remove estagnação do } \\
\text { tórax. }\end{array}$ \\
\hline (Tianliao)
\end{tabular}

\subsection{Análise estatística dos dados}

A normalidade das variáveis foi analisada empregando-se o teste de Kolmogorov-Smirnov; e a homogeneidade das variâncias, empregando-se o teste de Barttlet. Para comparações entre dois grupos, foi usado o teste t; diferenças de $\mathrm{p}<0,05$ foram consideradas significantes. As análises foram realizadas empregando-se o programa Prism 5 Software Package (GraphPad, USA, 2005). O teste de hipótese adotado foi o teste $t$-student com nível de confiança maior ou igual a $95.0 \%$, ou seja, $\mathrm{p}<0.05$.

\section{Resultados}

A Tabela 1 apresenta os dados com as características antropométricas do GE e GC. Foram aferidas as seguintes variáveis: idade, altura e peso. Com o intuito de verificar a homogeneidade dos grupos quanto as características antropométricas, procedeu-se uma compa- 
ração entre as variáveis acima citadas por meio do teste t. Os resultados foram idade $\mathrm{p}=0,15$ e peso $\mathrm{p}=0,74$, altura $\mathrm{p}=0,32$.

\begin{tabular}{lccc}
\multicolumn{4}{c}{ Tabela 1 - Caracterização dos dados antropométricos do grupo de } \\
estudo (GE) e grupo controle (GC). \\
\hline GE & Idade & Altura & Peso \\
Média & 22,14 & 1,68 & 79,57 \\
DP & 1,84 & 0,23 & 6,45 \\
GC & Idade & Altura & Peso \\
Média & 23,43 & 1,79 & 78,43 \\
DP & 1,13 & 0,08 & 6,45 \\
\hline
\end{tabular}

A Tabela 2 apresenta os dados referentes à resistência muscular localizada e a força de preensão palmar no GE e GC antes e depois a acupuntura e a condição de repouso. De forma geral, observa-se aumento no número de repetições nos dois grupos. Os resultados da força de preensão palmar revelaram aumento para o GE e redução para o GC, respectivamente. Ao aplicar os testes de hipótese para verificar diferença significativa entre o desempenho dos grupos antes e depois das condições testadas, notou-se que não houve aumento estatisticamente significativo para a resistência muscular localizada tanto no GE $p=0,39$ quanto no GC $p=0,51$. O aumento da força de preensão palmar no GE $p=0,45$ não foi estatisticamente significativo. A redução observada na força de preensão palmar no GC $p=0,50$ não se mostrou estatisticamente significativa.

Tabela 2 - Resistência muscular localizada e força de preensão palmar do membro dominante no grupo de estudo (GE) e grupo controle (GC).

\begin{tabular}{llllllllll}
\hline & Antes & Depois & Antes & & \multicolumn{3}{c}{ Depois } \\
GE & Rep & Rep & FPP 1 & FPP2 & FPP3 & FPP 1 & FPP2 & FPP3 \\
Média & 10,86 & 12,14 & 44,71 & 42,43 & 43,71 & 46,14 & 44,57 & 44,29 \\
DP & 2,85 & 2,54 & 6,89 & 6,75 & 7,01 & 5,27 & 5,44 & 5,70 \\
& & & & & & & & \\
& Antes & Depois & Antes & & & Depois & & \\
GC & Rep & Rep & FPP 1 & FPP2 & FPP3 & FPP 1 & FPP2 & FPP3 \\
Média & 13,29 & 14 & 48,86 & 51,43 & 52,00 & 49,43 & 48,00 & 49,43 \\
DP & 2,21 & 1,73 & 11,12 & 9,21 & 8,79 & 8,30 & 7,48 & 8,77 \\
\hline
\end{tabular}

\section{Discussão}

Por meio dos resultados obtidos, não foi possível avaliar a dimensão do efeito da acupuntura sobre o músculo, apesar de os dados da RML e FPP serem amplamente utilizados para estudar aumentos ou reduções da performance muscular.

A FPP é uma medida importante na mensuração da força total e correlaciona-se com outras variáveis como saúde, estado nutricional e aptidão física (BOHANNON, 1998; HANTEN et al., 1999). A variabilidade da força para sujeitos com idade entre 20 a 35 anos é de 44.1 a $63.6 \mathrm{~kg}$, sendo a média de 52,8kg. (BOHANNON et al., 2001). Os valores obtidos no presente estudo indicam que os voluntários encontram-se de acordo com a média geral. Apesar de a acupuntura não ter exercido melhora estatisticamente significativa sobre a dinamometria palmar, a FPP coletada demonstra a homogeneidade da amostra.

A resistência muscular localizada, segundo o American College of Sports Medicine (ACSM, 1998), fornece informações quanto ao estado funcional dos músculos testados. No presente estudo, observou-se que ambos os grupos aumentaram o número de repetições entre os testes de RML. Esses resultados foram contrários à hipótese inicial, que vislumbrava os seguintes senários: aumento da performance muscular no grupo com intervenção por acupuntura e redução da RML no grupo controle.

Nos exercícios calistênicos, utilizam-se, predominantemente, fibras do tipo I e IIa, enquanto no exercício resistido utilizam-se as fibras do tipo IIb. As fibras do tipo I são mais vascularizadas em relação as do tipo IIa e IIb. Essas últimas promovem contrações musculares vigorosas, com isso comprimem os vasos sanguíneos intra-musculares. Esse fato obriga as fibras musculares utilizarem vias bioenergéticas distintas. A fibra do tipo I usa a via aeróbica, já a do tipo IIa a via anaeróbica lática e a do tipo IIb anaeróbica alática (RASO et al., 2013).

A acupuntura tem ação importante no sistema nervoso. Graças a essa influência, a acupuntura tem efeito sistêmico, atuando, por exemplo, em órgãos efetuadores, glândulas e músculos (PELHAM et al., 2001).

Toda (2012) demonstrou que a acupuntura pode induzir o aumento da carnitina, responsável pela entrada de ácidos graxos na mitocôndria, reduzindo assim a fadiga muscular. Em seu estudo, dividiu ratos de laboratório em grupos tratamento e controle, sendo que o primeiro recebeu uma sessão de 15 minutos de acupuntura, o que 
resultou no incremento significativo dos níveis de carnitina no tecido muscular quando comparado ao grupo controle, que não sofreu nenhuma intervenção.

O ácido lático é resultante do sistema glicogênio-ácido lático, onde ocorre a glicólise, processo utilizado em períodos curtos a moderados de contração muscular (HALL, 2011). É um dos principais responsáveis pela fadiga, além de ser associado à diminuição da geração máxima de força (MCARDLE et al., 1996). A remoção do ácido lático é de extrema importância na recuperação pós-exercício do organismo (HALL, 2011).

A elevação do aporte sanguíneo é essencial durante o exercício físico, que requer grande demanda de oxigênio e nutrientes para a contração muscular (HALL, 2011). Sabe-se que a acupuntura pode estimular o aumento do volume sanguíneo no músculo e na pele (KUBO et al., 2010; SANDBERG et al., 2003) e a saturação de oxigênio no local da puntura (KUBO et al., 2010). Tsuchiya et al. (2007) demonstraram que, nas regiões corpóreas onde as agulhas foram aplicadas, ocorre elevação da taxa de óxido nítrico, um importante regulador do tônus vascular e fluxo sanguíneo, e por consequência há aumento da circulação sanguínea local. $\mathrm{O}$ aumento do fluxo sanguíneo pela acupuntura também pode estar relacionado à liberação de neuropeptídios vasodilatadores, provenientes das terminações nervosas aferentes ou eferentes parassimpáticas, como por exemplo, o calcitonin gene-related neuropeptide (CGRP) (SANDBERG et al., 2003).

A neurotensina é outro peptídeo capaz de causar vasodilatação e aumento da permeabilidade local, sendo também associada à analgesia (LUIZ et al., 2012). Em estudos com ratos, a neurotensina foi capaz de melhorar a analgesia por eletroacupuntura, sugerindo que houve favorecimento da liberação de encefalinas e $\beta$ endorfinas, e que a eletroacupuntura também pôde aumentar a secreção da neurotensina (BAI et al., 1999; LUIZ et al., 2012).

Diante disso, sugere-se que o tipo de RML adotada nesse estudo pode ter contribuído para menor efeito da acupuntura, uma vez que esse tipo de exercício exige maior recrutamento fibras muscular e assim maior adaptação neural, ou seja, é mais dependente da repetição do movimento em si, o que facilita o aprendizado motor e o consequente aumento de força. Já o RML usado nos outros estudos prioriza exercícios que dependem de um maior aporte sanguíneo para a região exercitada e, dessa forma, o mecanismo de aumento da perfusão nos locais punturados favorecem a continuidade do exercício, de- vido à remoção de catabólitos inibitórios da contração muscular e influxo de substratos para nutrição tecidual.

$\mathrm{O}$ argumento defendido acima tem sido apontado para explicar o insucesso da acupuntura em aumentar a força de preensão palmar de forma satisfatória nos outros estudos. O mecanismo de força de preensão palmar desfavorece a livre circulação sanguínea, pois o tipo de contração é isométrica, em que o músculo fica em estado de tensão aumentada continuamente durante a ação muscular, impedindo a dinâmica de contração e relaxamento. Esse evento faz com que os peptídeos produzidos via estimulação por acupuntura circulem nos músculos, diminuindo seu efeito.

O presente estudo apresentou como limitações a não existência do grupo placebo para comparação, entretanto, em todos os trabalhos com algum tipo de intervenção por acupuntura falsa ou placebo, os resultados da acupuntura verdadeira demonstraram que esta é estatisticamente mais eficaz em relação às anteriores (HÜBSCHER et al., 2010; MEDEIROS, 2009; SUN et al., 2009). Outras limitações se destacaram no estudo, por exemplo, a quantidade pequena de voluntários e variáveis ligadas ao desempenho esportivo como o uso de suplementos não foi controlada.

Aspectos psicológicos que possam colaborar negativamente com o desempenho atlético, como ansiedade, fadiga mental e estresse (YONGJUN et al., 2011) podem ser atenuados pelo uso de acupontos e de princípios da Medicina Tradicional Chinesa - MTC (ROSS, 2003). Apesar dos pontos utilizados possuírem funções energéticas relacionadas ao Qi e Xue (MARTINS, 2011), nesta pesquisa não foi realizada o diagnóstico energético dos voluntários, ou seja, fatores como desequilíbrio de Yin e Yang não foram levados em consideração, o que pode ter influenciado no resultado obtido. Ahmedov (2010), em sua revisão sobre acupuntura aplicada nos esportes e exercícios, comenta que, pelo fato de a acupuntura ser baseada no sistema da MTC, pode não funcionar adequadamente sem suas teorias e que o número de sessões também pode influir sobre os resultados.

Apesar das diversas preocupações envolvendo a acupuntura, a técnica tem ascendido no ocidente, sendo aplicada na promoção da saúde e bem-estar e, com frequência cada vez maior, direcionada para os esportes. $\mathrm{O}$ baixo custo, a simplicidade e os poucos efeitos adversos da acupuntura são incentivos para o emprego deste ramo da MTC em conjunto com as ciências da saúde. 


\section{Conclusão}

A acupuntura nesta pesquisa não se demonstrou eficaz para aumentar o rendimento dos sujeitos no teste de resistência muscular localizada e da força de preensão palmar. A realização de novos estudos incluindo grupos placebo, testes bioquímicos, imunológicos e hematológicos são de grande valia para melhor avaliar os efeitos da acupuntura no desempenho esportivo.

\section{Referências}

AHMEDOV, S. Ergogenic effect of acupuncture in sport and exercise: a brief review. Journal of Strenght and Conditioning Research, Champaign, v. 24, n. 5, p. 14211427, May 2010. doi: 10.1519/JSC.0b013e3181d156b1

\section{AMERICAN COLLEGE OF SPORTS MEDICINE} (ACSM). ACSM Position Stand: the recommended quantity and quality of exercise for developing and maintaining cardiorespiratory and muscular fitness, and flexibility in healthy adults. Medicine and Science in Sports and Exercises, Madison, v. 30, n. 6, p. 975-991, Jun. 1998.

ARAGÃO, J. C. B.; DANTAS, E. H. M.; DANTAS, B. H. A. Efeitos da resistência muscular localizada visando a autonomia funcional e a qualidade de vida do idoso. Fitness \& Performance Journal, v. 1, n. 3, p. 29-37, jul./set. 2002.

BAI, B. et al. Effect of anti-opioid peptid sera on the enhancement of electroacupuncture analgesia induced by neurotensine in PAG of rats. Sheng Li Xue Bau, Beijing, v. 51, n. 2, p. 244-248, abr. 1999.

BEMBEN, M. G. Isometric intermittent endurance of four muscle groups in men aged 20-74 yr. Medicine and Science in Sports and Exercise, Madison, v. 28, n. 1, p. 145-154, Jan. 1996.

BEMBEN, M. G. Age-related alterations in muscular endurance. Sports Medicine, Auckland, v. 25 n. 4, p. 259269, Apr. 1998. doi: 10.2165/00007256-199825040-00004

BOHANNON, R. W. Hand-grip dynamometry provides a valid indication of upper extremity strength impairment in home care patients. Journal of Hand Therapy, Philadelphia, v. 11, n. 4, p. 258-260, Oct./Dec. 1998. doi: 10.1016/S0894-1130(98)80021-5
BOHANNON, R.W. Dynamometer measurements of hand-grip strength predict multiple outcomes. Perceptual and Motor Skills, Missoula, v. 93, n. 2, p. 323-328, Oct. 2001. doi: 10.2466/pms.2001.93.2.323

DURWARD, B. R. et al. Movimento funcional humano: mensuração e análise. São Paulo: Manole, 2001.

FESS, E. E. Grip Strength. Clinical assessment recommendations. 2. ed. Chicago: American Society of Hand Therapistis, 1992.

HALL, J. E. Tratado de fisiologia médica. 12. ed. Rio de Janeiro: Elsevier, 2011.

HANTEN, W. P. et al. Maximum grip strength in normal subjects from 20 to 64 years of age. Journal of Hand Therapy, Philadelphia, v. 12, p. 193-200, Jul./Sep. 1999. doi: 10.1016/S0894-1130(99)80046-5

HÜBSCHER M. et al. Immediate effects of acupuncture on strength performance: a randomized, controlled crossover trial. European Journal of Applied Physiology, Berlin, v. 110, n. 2, p. 353-358, Sep. 2010. doi: 10.1007/ s00421-010-1510-y.

JAUNG-GENG, L.; SALAHIN, H. S.; JUNG-CHARNG, L. Investigation on the effects of ear acupressure on exercise-induced latic acid levels and the implications for athletic training. American Journal of Acupuncture, Capitola, v. 23, n. 4, p. 309-313, Oct./Dec. 1995.

KUBO, K. et al. Effects of acupuncture and heating on blood volume and oxygen saturation of human Achilles tendon in vivo. European Journal of Applied Physiology, Berlin, v. 109, p. 545-550, Jun. 2010. doi: 10.1007/ s00421-010-1368-z.

LIAN, Y. et al. Atlas gráfico de acupuntura: um manual ilustrado dos pontos de acupuntura. São Paulo: Konemann, 2005.

LO, Y. L.; CUI, S. L.; FOOK-CHONG, S. The effect of acupuncture on motor cortex excitability and plasticity. Neuroscience Letters, Limerick, v. 384, n. 1-2, p. 145149, Aug. 2005. doi:10.1016/j.neulet.2005.04.083 
LUIZ, A. B.; BABINSKI, M. A.; FERREIRA, A. S. Neurobiologia da analgesia induzida por acupuntura manual $\mathrm{e}$ eletroacupuntura: revisão de literatura. Journal of Naturology and Complementary Therapies, Florianópolis, v. 1, n. 1, p. 71-84, jan./dez. 2012.

MACIOCIA, G. Os fundamentos da medicina chinesa: um texto abrangente para acupunturistas e fitoterapeutas. São Paulo: Roca, 1996.

MARTINS, E. I. S. Atlas dos pontos de acupuntura: guia de localização. São Paulo: Roca, 2011.

MCARDLE, W. D.; KATCH, F. I.; KATCH, V. L. Fisiologia do exercício: energia, nutrição e desempenho humano. 4. ed. Rio de Janeiro: Guanabara Koogan, 1996.

MEDEIROS, R.; SAAD, M. Acupuntura: efeitos fisiológicos além do placebo. O Mundo da Saúde, São Paulo, v. 33 , n. 1, p. 69-72, jan. 2009.

MOREIRA, D.; GODOY, J. R. P.; SILVA JÚNIOR, W. Estudo sobre a realização da preensão palmar com a utilização do dinamômetro: considerações anatômicas e cinésiológicas. Fisioterapia Brasil, Rio de Janeiro, v. 2, n. 5, p. 295-300, set./out. 2001.

MOREIRA, D.; GODOY, J. R. P.; SILVA JÚNIOR, W. Abordagem sobre preensão palmar utilizando o dinamômetro JAMAR: uma revisão de literatura. Revista Brasileira de Ciência e Movimento, Brasília v. 11, n. 2, p. 95-99, jun. 2003.

PELHAM, T. W.; HOLT, L. E.; STALKER, R. Acupuncture in human performance. Journal of Strenght and Conditioning Research, Champaign, v. 15, n. 2, p. 266-271, May. 2001.
RASO, V.; GREVE, J. M. D.; POLITO, M. D. Pollock: fisiologia clínica do exercício. Barueri: Manole, 2013.

ROSS, J. Combinações dos pontos de acupuntura: a chave para o êxito clínico. São Paulo: Roca, 2003.

SANDBERG, M. et al. Effects of acupuncture on skin and muscle blood flow in healthy subjects. European Journal of Applied Physiology, Berlin, v. 90, n. 1-2, p. 114-119, Sep. 2003. doi: 10.1007/s00421-003-0825-3

SUN, D.; ZHANG, Y.; CHEN, D. Research progress in sports fatigue prevented and treated by acupuncture. Journal of Acupuncture and Tuina Science, Shangai, v. 7, n. 2, p. 123-128, Apr. 2009. doi: 10.1007/s11726-0090123-7

TODA, S. Effect of acupuncture on carnitine for skeletal muscle fatigue. Scientific Research, Delaware, v. 3, n. 1, p. 9-12, Mar. 2012. doi: 10.4236/cm.2012.31003

TSUCHIYA, M. et al. Acupuncture enhances generation of nitric oxide and increases local circulation. Anesthesia \& Analgesia, Cleveland, v. 104, n. 2, p. 301-307, Feb. 2007. doi: 10.1213/01.ane.0000230622.16367.fb

WHITE, P. et al. Meeting report: standardization of nomenclature in acupuncture research (SoNAR). Evidence Based Complementary and Alternative Medicine, $\mathrm{Ox}$ ford, v. 4, n. 2, p. 267-270, Jun. 2007. doi: 10.1093/ecam/ nel095

YONGJUN, S.; XIUFENG, W. Self-regulation of elite athletes in China. Social Behavior and Personality, New Zealand, v. 39, n. 8, p. 1035-1044, Oct. 2011. doi: 10.2224/ sbp.2011.39.8.1035 